\title{
Psychological therapies for post-traumatic stress
}

\section{disorder ${ }^{\dagger}$}

\author{
GWEN ADSHEAD
}

\section{Background After exposure to traumatic stressors, a subgroup of survivors (20-30\%) will develop post-traumatic stress disorder (PTSD).}

\begin{abstract}
Aims Since the incidence and prevalence rates for PTSD in the community are significant, it is important that general practitioners and psychiatrists be familiar with possible therapeutic options. In this review we shall look at the published evidence about the effectiveness of psychological treatments for PTSD.
\end{abstract}

\section{Method The psychopathological mechanisms involved in PTSD are discussed. Studies of the effectiveness of different psychological therapies are reviewed.}

\section{Results The review suggests that persistent fear or shame reactions are key aspects of PTSD. Evidence from systematic reviews suggests that psychotherapeutic treatments are effective in the therapy of reactions based on fear, and may increase the effectiveness of pharmacological therapy. There is less systematic evidence for the efficacy of interventions for symptoms based on shame.}

Conclusions Althougha proportion of patients with complex or chronic PTSD may require specialist interventions, most patients can be treated effectively by a general psychiatric service which can offer both pharmacological and psychological interventions.

Declaration of interest None.

†See editorial, pp.93-94, this issue.
It is clear that traumatic events, especially those which induce significant feelings of fear and helplessness, can cause psychological disorders in those who survive them. The most common post-traumatic disorders are depression and substance misuse; other possible disorders include acute stress reactions, anxiety states such as phobic and panic disorders, and personality change after trauma. It is unusual for any one post-traumatic disorder to occur in isolation; comorbidity is the norm.

Although one of the less common types of post-traumatic psychopathology, posttraumatic stress disorder (PTSD) has received considerable clinical and research attention over the past 18 years. PTSD occurs in $20-30 \%$ of people who are exposed to traumatic stressors; it is rare for patients to present with 'pure' PTSD.

It is not possible in a brief review to examine the indications for, or efficacy of, psychological therapies for all the posttraumatic disorders. For most disorders, standard therapeutic interventions are indicated; and therefore this review will be confined to psychological therapies for PTSD in adults. The treatment of PTSD in children is a specialist area which will not be dealt with here. Although psychopharmacological treatments are outside the remit of this review, they are often combined with psychological interventions, and play an important role in the effective treatment of PTSD. Recent research demonstrates that PTSD is a neurophysiological disorder, with demonstrable effects on the hypothalamic-pituitary axis, hippocampal volume, and endogenous opioid function.

\section{ASSESSMENT FOR PSYCHOLOGICALTHERAPY OF PTSD}

Clinicians are as likely to see people with PTSD as they are to see patients with schizophrenia. The prevalence of PTSD is $1 \%$ in the general (US) population; lifetime prevalence rates are reported to be up to 9.2\% (Helzer et al, 1987; Kessler et al, 1995). The prevalence is higher in those exposed to traumatic events; the experience of interpersonal violence increases the prevalence up to $20-30 \%$. Treatment planning entails a detailed assessment addressing the key features of PTSD.

The entry criterion (Criterion A) for the diagnosis of PTSD (DSM-IV; American Psychiatric Association, 1994) defines traumatic stressors (as opposed to ordinarily unpleasant stressors) as the witnessing of, or experiencing, threat to life or severe injury to the self, or to a significant other. This objective aspect of Criterion A reflects the established finding that threat to life is a potent predictor of PTSD, which distinguishes PTSD from other types of stressinduced illness. The other specifically traumatic aspect of stressors is the subjective experience of "intense fear and helplessness". The experience of a lifethreatening stressor, and the experience of intense fear and/or helplessness, are necessary for a diagnosis of PTSD.

The objective and subjective criteria for PTSD neatly reflect current thinking about the aetiology of PTSD. One the one hand, the risk of developing PTSD is directly related to exposure to traumatic stressors; that is, the more exposure, the higher the risk of developing PTSD. The duration of the traumatic event is important as an indicator of the degree of exposure, and the assessor needs to enquire about the 'fear' aspect of the traumatic experience, especially the perception of threat to life.

On the other hand, there is ample evidence that personality structure and experience (such as neuroticism or childhood adversity) in the period before the traumatic event also influence the risk of developing PTSD. The personal history of the individual patient needs to be examined for information about previous vulnerability factors (such as early fear experiences and insecure attachments) and resilience factors (such as self-esteem). Early fear experiences may be significant in terms of later capacity to manage threat (Dodge et al, 1997). Individuals may have high degrees of resilience even where there has been early and severe childhood adversity.

When the trauma occurred is therapeutically relevant. If the traumatic event occurred between 4 and 8 weeks before 
the interview, then the patient is likely to be experiencing severe symptoms. If many dissociative symptoms are present, these may constitute an acute stress disorder (American Psychiatric Association, 1994). Although such disorders often resolve spontaneously over the following 4-6 weeks, patients may benefit from a shortterm intervention (see below). Acute stress disorder is a risk factor for the development of later PTSD.

The nature of the patient's traumatic experience is also important to assess, because some types of trauma are more likely than others to provoke PTSD. Highrisk stressors include those which involve grotesque sensory images, interpersonal violence (especially sexual assault) and chronic fear and helplessness.

Finally, it is important to assess the patient's world view (Weltanschauung) before the trauma occurred. Individuals at risk of developing PTSD include those who tend to have an 'all or nothing' view of events and their part in them. Cultural stereotypes (especially about gender role) are highly relevant (Turner et al, 1996). Resilient individuals tend to see themselves as neither omnipotent nor helpless in the face of stress; and they are not ashamed to seek help and use what is offered. In contrast, vulnerable individuals tend to see care-seeking as shameful and anxietyinducing.

\section{TREAT MENT PLANNING}

The conscious and/or unconscious neurophysiological sequelae of experiencing traumatic stressors are overlaid with the psychological meaning ascribed to the experience by the individual, and his or her conscious and unconscious beliefs about him or herself and the world. The sudden and acute experience of terror, and one's own mortality, has an impact which is both physiological and psychological. It is therefore essential to consider both these aspects in treatment planning.

Most explanatory models of PTSD have argued that it represents a failure to process the experience of fear; reflecting in turn either a previous vulnerability to fear, or exposure to extremes of fear (Foa \& Kozak, 1986). However, some patients suffer more from other affects, especially sadness, guilt and shame (Andrews, 1998). For such individuals, it seems likely that something about the traumatic experience affected their sense of themselves: the way they think about themselves, and about others in relationships with them. Their symptoms seem to express less about the shattering of external security by fear, and more about a fragmentation of an inner sense of security.

These different types of affective reactions have implications for psychological therapy. Patients with principally fearbased reactions will need therapies which address the management of fear and anxiety. Patients with shame-based reactions will need a psychological therapy which focuses on cognitive representations of the self, and relationships with others. Previous experiences of fear and safety will be relevant to both types of patient, especially in terms of forming a therapeutic alliance. Both shame and fear reactions can coexist in the same patient.

\section{REVIEW OF PSYCHOLOGICAL THERAPIES FOR PTSD: META-ANALYSES}

This paper is not a comprehensive systematic review, although we searched the literature from the past 5 years both by computer and 'by hand'. Two recent meta-analyses have studied the psychological treatment of PTSD (Sherman, 1998; van Etten \& Taylor, 1998). Studies using psychodynamic methods were included in both analyses. Psychological therapies appear to be more effective than psychotropic medication (van Etten \& Taylor, 1998), although patients treated with both improved more than did controls. Sherman found significant effects for all psychological therapies, particularly for behavioural therapy, but found no support for one single rationale for therapy. His positive view contrasts with that of Shalev et al (1996), who found less significant effects for psychological therapies. However, Shalev et al included several studies that were open-ended, and where the therapy programme was less structured.

\section{THER APIES ADDRESSING FEAR}

Most of the therapies described in the metaanalyses include behavioural and cognitive strategies for managing fear by exposure to the feared stimulus, whether in imagination or in the external world. Exposure therapy seems to be effective for many patients, including combat veterans and victims of interpersonal violence. Exposure therapy usually takes the form of systematic desensitisation, in which the patient is gradually encouraged to reduce avoidance of the feared image or memory. Exposure may be imaginary or live (Richards et al, 1994), and utilises trauma-based imagery generated by the patient in the form of remembered images, or a written script.

Exposure therapies can also be combined with cognitive processing interventions (e.g. Resick \& Schnicke, 1993), stress inoculation and relaxation techniques, and anxiety management training (Rothbaum \& Foa, 1996). Both exposure and cognitive restructuring techniques seem to be effective, and are more effective than relaxation alone (Marks et al, 1998). Another form of exposure therapy employs cognitive reprocessing combined with saccadic eye-movements (eye-movement desensitisation and reprocessing, EMDR). Recent studies suggest that this strategy can be effective with combat veterans, and survivors of child abuse and disasters.

Most of the studies of fear-based therapies report a decrease in 'target' symptoms of PTSD, namely avoidance and intrusion phenomena such as flashbacks. Fear-based therapies are probably most effective in patients: who describe high levels of intrusive phenomena; who are not severely depressed or misusing substances; in whom the traumatic experience was a relatively discrete episode in their lives; who do not have extensive histories of trauma previous to the index event; and who had reasonably good psychological health before the index event.

The traumatic event need not be recent for fear-based therapies to be effective, as is demonstrated by Case 1 .

\section{Case I. Mrs Jenkins}

Several years before presentation, Mrs Jenkins had been traumatically bereaved while on holiday abroad. Her husband was killed by a bomb which exploded outside their hotel during a period of civil unrest. Mrs Jenkins was able to give an account of what had happened (hearing the noise, coming out and finding the body), but it was lacking in detail. She was drinking heavily, socially withdrawn, and sensitive to loud noises in the street; fireworks night was especially difficult. After some initial work to establish the therapeutic alliance, exposure work was planned. Mrs Jenkins agreed to describe her experiences in as much detail as possible, over a number of sessions. She was also encouraged to write an account of her experience between sessions. At the third session, Mrs Jenkins recalled afresh seeing the "blueness" of her husband's eyes as 
he lay dying. This memory was acutely distressing to recall, but significant symptomatic relief followed this session. Subsequent sessions were easier, and at the end of six sessions, Mrs Jenkins was clinically improved and ready to be discharged.

Most therapy now takes place in outpatient settings. It is relatively short-term (8-15 sessions), and patients are encouraged to do 'homework' between sessions. The session content may follow a set order according to established protocols. Although cognitive techniques do address the question of dysfunctional beliefs and self-schemata, most of the emphasis of these therapies is on the minimisation of avoidance behaviour, and experience of avoided fear, with subsequent reduction of anxiety and increase in confidence.

Much of the earliest work in this area was done with combat veterans, and in in-patient settings. Typically such work included group psychotherapy and behavioural interventions. In a review of such interventions, Read Johnson (1997) suggests that the in-patient programmes were ineffective for PTSD. However, a related discussion paper points out that nearly half of the subjects studied had histories of childhood trauma, and many had experienced significant traumatic experience after combat. Short-term or unimodal therapeutic strategies are unlikely to be successful with patients who need to make sense of both fear and shame experiences, and who are having to cope with many other stressors simultaneously.

\section{THERAPIES ADDRESSING \\ SHAME}

The experience of shame is closely linked to the feelings of depression, guilt and humiliation. Shame and guilt can be difficult to distinguish; although both involve negative self-evaluation, feelings of guilt may relate more to behaviour, whereas shame may reflect low self-esteem and self-criticism (Andrews, 1998). Patients with posttraumatic shame experiences tend to describe less intrusion and re-experiencing phenomena associated with PTSD. Instead, they describe experiences of ruminating on what went wrong, how they failed, their sense of hopelessness and helplessness, and other self-directed cognitions such as guilt. The painful thoughts and feelings seem to be less to do with fear of death, and more to do with the loss of an inner sense of self-confidence and esteem.
This type of post-traumatic presentation, which particularly affects the patient's concept of self, has also been called 'complex PTSD' (Herman, 1992). Herman describes the following characteristics of complex PTSD:

(a) experience of prolonged abusive coercion;

(b) alterations in affect regulation;

(c) alterations in consciousness, especially depersonalisation and de-realisation states;

(d) alterations in self-perception;

(e) alterations in perception of trauma/ perpetrator;

(f) alterations in relations with others; and

(g) alterations in systems of meaning.

In DSM-IV field trials, Roth et al (1997) found that complex PTSD is associated with the experience of prolonged exposure to trauma (i.e. over days, months or years), and first exposure at an early age. Complex PTSD is also associated with prolonged interpersonal victimisation, such as battering by a partner, torture or prisonerof-war experiences, and child abuse.

An atypical case makes a good example (and also makes the point that complex PTSD is not only related to child abuse or torture).

\section{Case 2. Mr Smith}

Mr Smith was assaulted from behind by a man whom he could not see, and who was unknown to him. He was repeatedly beaten about the head, and the assault only ended when the man ran off. Mr Smith was powerless to defend himself against this man. $\mathrm{He}$ felt helpless and humiliated. This experience contrasted with his previous views about himself as a powerful man, who could handle anything and was the leader and provider for his family.

Mr Smith's self identity was bound up with being strong and powerful. Thus, at one level he identifies strongly with his assailant, shares his values and wants to be like him. He despises his own experience of being assaulted and helpless. On the other hand, to value his victimised self, he must make a split from his assailant in his mind, and let that identification go. He has to lose and mourn a real part of himself. $\mathrm{He}$ alternates between feeling ashamed and depressed.

It is perhaps noteworthy that Mr Smith's difficulties developed after an attack which lasted some 20 minutes. One can only speculate about the damage done to the sense of self, if attacks like this go on for many years. Although the symptoms of complex PTSD resemble depressive symptoms, the alteration in the sense of self is crucial to personal functioning. It may be that the concept of complex PTSD overlaps with the concept of "enduring personality change after trauma", as described in ICD-10 (World Health Organization, 1992).

The aim of shame-based therapies, especially in cases of complex PTSD, is to try to help the patient to restore some sense of meaning in terms of their own sense of self, and to move from the traumatic sense of self to a narrative (and developing) sense of self (Lindy, 1996). Sessions focus on beliefs about the self and others and on the experience of painful feelings such as shame and rage. The awareness and expression of the patient's anger are important aspects of the therapeutic process; for many patients, a continuing sense of shame relates to a profound and pervasive anger. In such cases, there may be less need to go over, or rehearse, details of the traumatic experience: indeed, such a process may be counter-productive because it can increase distress and shame in someone who is already not coping well with such feelings. In general, the patient is not avoiding thinking about the experience, but rather is ruminating about it. Exposure therapy (including detailed rehearsal or exposure to traumatic memory) is therefore unlikely to be helpful in such cases, because it dismantles defences against psychological distress. Instead, the therapeutic emphasis is on support for coping strategies, and the shoring-up of defences (Embry, 1990). The therapeutic aim is to generate with the patient an environment in which feelings of shame (and sometimes of accompanying rage) can be safely explored. Although it is vital for both fear-based and shame-based therapies that the therapeutic environment is safe (Turner et al, 1996), this aspect of treatment is more pronounced for patients with complex PTSD because they lack inner capacities for self-soothing, at least initially. The therapist may have to help the patient to learn to soothe themself, and teach them how to contain arousal; adjunctive medication can be useful here.

\section{Difference between fear-based and shame-based therapies}

Unlike fear-based therapies, in a shamebased therapy the relationship between the patient and the therapist is likely to be itself a major part of the therapeutic process. This is because, for many patients, the traumatic events took place in the context 
of an ongoing relationship; the relationship was the trauma. Therefore, the patient's thoughts and fantasies about the therapist (transference) and the therapist's thoughts and fantasies about the patient (countertransference) will become part of the clinical effort. Patients may find it hard to trust the therapist, and the establishment of a therapeutic alliance may take months rather than weeks. Alternatively, patients may become intensely dependent on their therapist, and seek to hand over to them all responsibility for their health.

The therapist, too may have responses to the patient which need consideration. In particular, the therapist needs to guard against the following countertransferential reactions:

(a) Rejection of the patient ("'they're too awful") - such a feeling may reflect the clinician's own reluctance to think about terrible experiences.

(b) Certainty for the patient ("let me tell you what happened to you") - this may represent a way for the therapist to control anxiety-provoking material. Other types of control reaction in the therapist include giving advice or instructions to the patient.

(c) Exploitation of the patient's compliance or dependence ("you don't mind if I do this, do you?").

A potent way to reduce psychological shame is to decrease the sense of alienation and isolation that it brings. It may be for this reason that group therapies have been widely used in post-traumatic psychotherapy (Turner et al, 1996). These can be short-term or long-term; exclusively focused on a traumatic event or not. Many patients have found that the development of self-help groups is effective not only in reducing shame but also in increasing a sense of self-empowerment, challenging passivity and helplessness. The main role of the therapist (where there is one) will be to establish safety in the group, with particular attention to group boundaries and setting limits to behaviour.

Acute readers may have noticed a similarity between the psychotherapy of complex PTSD and the psychotherapeutic treatment of personality disorders. This is unsurprising, given that early childhood trauma is an established risk factor for the development of later personality disorder. Clinicians working with personalitydisordered patients may find some useful information in the literature on the

\section{CLINICAL IMPLICATIONS}

PTSD can be treated effectively with psychological interventions.

- Psychological interventions are more effective than pharmacological interventions, although both are better than placebo.

Fear-based reactions and shame-based reactions may need different types of psychological therapy.

\section{LIMITATIONS}

- There have been far fewer studies of the efficacy of psychological therapies for complex PTSD, or shame-based reactions.

- There may be other psychopathological variables active, apart from shame and fear.

- Most treatment studies are made on highly selected samples.

GWEN ADSHEAD, MRCPsych, Traumatic Stress Clinic, 73 Charlotte Street, London WIP ILB, or Psychotherapy Department, Broadmoor Hospital, Crowthorne, Berkshire RG45 7EG

(First received 8 December 1998, final revision 30 November 1999, accepted 7 December 1999)

psychodynamic treatment of PTSD (Embry, 1990; Lindy, 1996).

\section{EARLY AND BRIEF INTERVENTIONS}

Although it was hoped that early interventions such as debriefing might prevent the development of PTSD, there is little evidence that this is so (Wessely et al, 1997; Bolwig, 1998). In fact, there is some reason to think that some vulnerable individuals may experience more symptoms as a result of a debriefing intervention (Bisson et al, 1997). Such data suggest that early interventions may only be of value to some individuals, and that such interventions need to be targeted. When supportive interventions are offered to survivors of traumatic events, most people do report finding them helpful, so there is still research to be done on what this 'help' might be. There are important implications for public health and civil litigation here.

\section{CLINICAL IMPLICATIONS}

All clinicians need to know something about the psychotherapeutic treatment of PTSD; not least because epidemiological research suggests that its prevalence in the community is high. There is every reason to think that many patients who are referred for psychiatric help may be suffering from one of the post-traumatic stress disorders. Although there are now a number of specialised treatment centres for psychological trauma in the UK, many people can, and should, be treated by their home-based psychiatric and psychological services.

\section{ACKNOWLEDGEMENTS}

I am grateful to my colleagues at the Traumatic Stress Clinic, Middlesex Hospital, and to Dr Gill Mezey and Dr Bernice Andrews for their help with the writing of this paper. I am also grateful for the comments of two anonymous reviewers, and for the secretarial assistance of Anne Kavanagh.

To preserve anonymity, the cases described are composites of real cases and cannot be identified with any individual.

\section{REFERENCES}

American Psychiatric Association (1994) Diagnostic and Statistical Manual of Mental Disorders (4th edn) (DSM-IV). Washington, DC: APA.

Andrews, B. (1998) Shame and childhood abuse. In Shame: Interpersonal Behaviour, Psychopathology and 
Culture (eds P. Gilbert \& B. Andrews), pp. 176-190. Oxford: Oxford University Press.

Bisson, J. I., Jenkins, P. L., Alexander, J., et al (1997) Randomised controlled trial of psychological debriefing for victims of acute burn trauma. British journal of Psychiatry, 17I, 78-81.

Bolwig, T. (1998) Debriefing after psychological trauma. Acta Psychiatrica Scandinavica, 98, 169-170.

Dodge, K. A., Pettit, G. \& Bates, J. E. (1997) How experience of early physical abuse leads children to become chronically aggressive. In Developmental Perspectives on Trauma: Theory, Research and Intervention (eds D. Cicchetti \& S. Toth), pp. 263-288. Rochester, NY University of Rochester Press.

Embry, C. (1990) Psychotherapeutic interventions in chronic post-traumatic stress disorder. In Posttraumatic Stress Disorder: Etiology, Phenomenology and Treatment (eds M.Wolf \& A. Mosnaim), pp. 226-237. Washington, DC: American Psychiatric Press.

Foa, E. \& Kozak, M. (1986) Emotional processing of fear: exposure to corrective information. Psychological Bulletin, 99, 20-35.

Helzer, J. E., Robins, L. N. \& McEvoy, L. (1987) Posttraumatic stress disorder in the general population. New England Journal of Medicine, 317, 1630-1634.

Herman, J. L. (1992) Trauma and Recovery. New York: Basic Books.

Kessler, R. C., Sonnega, A., Bromet, E., et al

(1995) Post-traumatic stress disorder in the National
Co-Morbidity Survey. Archives of General Psychiatry, 52 , 1048-1060.

Lindy, J. (1996) Psychoanalytic psychotherapy of posttraumatic stress disorder: the nature of the therapeutic relationship. In Traumatic Stress: The Effects of

Overwhelming Experience on Mind, Body and Society (eds B. van der Kolk, A. McFarlane \& L. Weisaeth), pp. 525-536. New York: Guilford.

Marks, I., Lovell, K., Noshirvani, H., et al (1998) Treatment of post-traumatic stress disorder by exposure and/or cognitive restructuring. Archives of General Psychiatry, 55, 317-325.

Read Johnson, D. (1997) Introduction: inside the specialised inpatient PTSD units of the Department of Veterans Affairs. Journal of Traumatic Stress, 10, 357-360.

Resick, P. A. \& Schnicke, M. K. (1993) Cognitive Processing Therapy for Rape Victims: A Treatment Manual. London: Sage.

Richards, D., Lovell, K. \& Marks, I. (1994) Posttraumatic stress disorder: Evaluation of a behavioura treatment programme. Journal of Traumatic Stress, 7 669-680.

Roth, S., Newman, E., Pelcovitz, D., et al (1997) Complex PTSD in victims exposed to sexual and physical abuse: results from the DSM-IV field trial for Posttraumatic Stress Disorder. Journal of Traumatic Stress, 10 539-556.

Rothbaum, B. O. \& Foa, E. (1996) Cognitivebehavioural therapy for posttraumatic stress disorder. In
Traumatic Stress: The Effects of Overwhelming Experience on Mind, Body and Society (eds B. van der Kolk, A. McFarlane \& L.Weisaeth), pp. 49I-509. New York: Guilford.

Shalev, A., Bonne, O. \& Eth, S. (1996) Treatment of post-traumatic stress disorder: a review. Psychosomatic Medicine, 58, 165-182.

Sherman, J. J. (1998) Effects of psychotherapeutic treatments for PTSD: a meta-analysis of controlled trials. Journal of Traumatic Stress, II, 4I3-436.

Turner, S., McFarlane, A. \& van der Kolk, B. (1996) The therapeutic environment and new explorations in the treatment of post-traumatic stress disorder. In Traumatic Stress: The Effects of Overwhelming Experience on Mind, Body and Society (eds B. van der Kolk, A. McFarlane \& L.Weisaeth), pp. 537-558. New York: Guilford.

van Etten, M. L. \& Taylor, S. (1998) Comparative efficacy of treatment for post-traumatic stress disorder: a meta analysis. Clinical Psychology \& Psychotherapy, 5, 126-144.

Wessely, S., Rose, S. \& Bisson, J. (1997) A systematic review of brief psychological interventions ("debriefing") for the treatment of trauma-related symptoms and the prevention of post-traumatic stress disorder. Cochrane Review 25/11/97. Oxford: Update Software.

World Health Organization (1992) The ICD-10 Classification of Mental and Behavioural Disorders. Geneva: WHO. 